\title{
Padres, hijos e internet. Socialización familiar de la red*
}

\section{Parents, Children and Internet Use. Family Socialization on the Internet}

Recepción: 09 Noviembre 2015 | Aprobación: 27 Junio 2017

\author{
Santiago Yubero \\ Universidad de Castilla-La Mancha, España \\ ORCID: http://orcid.org/0000-0002-7148-7958 \\ ELISA LARRAÑAGA \\ Universidad de Castilla-La Mancha, España \\ Raúl NaVARro \\ Universidad de Castilla-La Mancha, España \\ María Elche \\ Universidad de Castilla-La Mancha, España
}

a Autor de correspondencia. Correo electrónico: santiago.yubero@uclm.es

Para citar este artículo: Yubero, S., Larrañaga, E., Navarro, R., \& Elche, M. (2018). Padres, hijos e Internet. Socialización familiar de la Red. Una relación compleja. Universitas Psychologica, 17(2), 1-13. https://doi.org/10.11144/Javeriana.upsy172.phis

\section{RESUMEN}

Estudios previos muestran resultados dispares en relación con la efectividad de la mediación parental en el uso de Internet. El objetivo fue analizar la influencia de los padres sobre el empleo de la red que llevan a cabo los hijos. Participaron en el estudio 938 familias con hijos entre 12 y 17 años. El análisis de regresión destaca la importancia del modelaje de los padres. Los resultados obtenidos muestran que la acción de los padres sobre el empleo de Internet de los hijos está mediada por el empleo que ellos mismos realizan de Internet. Este estudio apunta a la necesidad de que los padres se impliquen en los procesos de socialización sobre el uso de Internet.

Palabras clave

mediación familiar; socialización; empleo Internet de los padres; uso adolescente de Internet.

\section{ABSTRACT}

Previous research has shown mixed results regarding the effectiveness of parental mediation in the Internet use. The main purpose of this study was to analyze the influence of parents on adolescent's Internet use. Sample consisted in 938 Spanish families with adolescents aged between 12-to-17 years old. Regression analysis confirmed the relationship between adolescents' Internet use and family variables, emphasizing the importance of modeling performed by parents. Specifically, results showed that the effect of parents' actions over their children's Internet use is mediated by the parent's use of the Internet. This study points out the need for parents to be involved in the socialization processes related with Internet use.

Keywords

family mediation; socialization; parents' Internet use; Adolescents' Internet use. 
En los últimos años, se ha producido un incremento espectacular del uso de las tecnologías de la información y de Internet, principalmente entre la población más joven (Estévez, Bayón, de la Cruz, \& FernándezLiria, 2009; Holtz \& Appel, 2011; Viñas, 2009). Fosk (2010) informó que en el final de la pasada década, Internet también había penetrado fuertemente en América Latina. Así mismo, con este incremento se ha detectado la existencia de conductas de riesgo, como arreglar encuentros con desconocidos o dar datos personales (Fernández-Montalvo, Peñalva, \& Irazabal, 2014), comportamientos de abuso en el empleo (Osma, 2002), e incluso adicción (Ruiz-Olivares, Lucena, Pino, \& Herruzo, 2010). Por otra parte, Internet también tiene efectos positivos. Heyam (2014), a través de un metaanálisis, concluye que el empleo de las redes sociales facilita la comunicación, la socialización y la colaboración. El empleo de Internet también incide positivamente sobre el éxito académico (Torres, Duan, Gómez-Alvarado, Marín-Gutiérrez, Segarra, 2016). Gil-Flores (2009), con estudiantes de secundaria, encontró relación significativa entre la frecuencia del empleo de los ordenadores y las calificaciones en matemáticas y comunicación lingüística. La búsqueda de información en la web también mejora las calificaciones (Chen \& Fu, 2009; Leung \& Lee, 2012). Mendivelso, Méndez, y Ramírez-Martinell (2017), en una experiencia con recursos educativos tecnológicos, en estudiantes de Boyacá, Colombia, entre 10 y 13 años, demostraron que la tecnología despertaba el interés de los alumnos por la materia.

Estamos de acuerdo con Parra (2010), en que no puede considerarse al internet como un medio directamente perjudicial, pero tampoco exclusivamente positivo. Como afirma GarcíaPiña (2008, p. 273), "es conveniente y necesaria, la presencia de un adulto durante esta actividad". El papel de los padres en la socialización de la Red es básico para alcanzar un empleo responsable de este nuevo medio de interacción social.

Los estudios muestran tasas superiores al 90 \% de jóvenes conectados a Internet (García,
López de Ayala, \& Catalina, 2013; Hernández, Robles, \& Mella, 2009; Valkenburg \& Peter, 2007), con un empleo vinculado principalmente a las relaciones sociales (Arango, Bringué, \& Sádaba, 2010; Flores, 2009; Herrera-Batista, 2009). Schwarz (2011) sugiere, incluso, que los jóvenes se han alejado de la interacción cara a cara o por teléfono, hacia la comunicación basada en la mensajería instantánea. Almansa, Fonseca, y Castillo (2013), a través de metodología cualitativa, confirmaron que las redes sociales se han convertido en el principal ámbito de interacción social entre los jóvenes españoles y colombianos. Los chicos también acceden en gran medida para jugar online (Arango et al., 2010; Fernández-Montalvo et al., 2014).

Todos los menores, en mayor o menor medida, acceden a Internet en sus casas. En Latinoamérica, lo hacen entre $50 \%$ y el 70 \%, según el país (Arango et al., 2010; HerreraBatista, 2009; Parra, 2010). En España, Bringué, Sábada, y Tolsa (2011) informan que el 89 \% de los adolescentes navega desde su casa, situación que sitúa a los padres en un espacio privilegiado de actuación (García-Piña, 2008). Sin embargo, el avance vertiginoso de las tecnologías ha abierto una brecha digital entre adultos y jóvenes (Thurlow \& McKay, 2003). Muchos padres se preocupan cuando ven a sus hijos con las TIC, ante la pantalla del ordenador o un teléfono móvil, conectados a las redes virtuales (Echeburrúa, Labrador, \& Becoña, 2009; Parra, 2010).

Los adultos incorporan a los nuevos miembros a la cultura de su entorno a través del proceso de socialización, que consiste en la adquisición de los hábitos, las normas y los valores característicos de la cultura donde debe insertarse el individuo. La familia representa el primer contexto social que acoge al individuo e interviene, en primera instancia, en su inmersión dentro de un contexto sociocultural específico. Es el primer agente de transmisión cultural, y permanece durante toda la vida del individuo. Se trata de un proceso gradual, secuenciado, sincronizado con el desarrollo del individuo, y que implica una participación activa a través del contacto social continuado. Los padres ejercen 
su papel socializador, fundamentalmente por dos vías de actuación: como modelos (acción de modelaje, actitud ante Internet) y mediante las prácticas educativas que llevan a cabo (acciones de mediación). En el proceso de socialización familiar, Internet también debe ocupar un espacio importante (Castellana, Sánchez-Carbonell, Graner, \& Beranuy, 2007; Dehue, Bolman, \& Völlink, 2008). La mediación parental es un concepto que hace referencia a las actividades llevadas a cabo por los padres para proteger a sus hijos de los peligros que conlleva Internet (Mesh, 2009), y que podemos agrupar en tres categorías: la mediación restrictiva, que supone limitar la cantidad de tiempo que los adolescentes se conectan y las páginas a las que acceden, es restrictiva porque el adolescente no participa en la decisión, sino que es tomada por sus padres; la mediación activa, que hace referencia a la discusión de temas relacionados con el uso de Internet y la creación conjunta de normas; y el uso conjunto, en el que los padres están presentes mientras el adolescente está conectado a Internet.

Son varios los estudios que han analizado la influencia de los padres sobre el uso de la Red. La mayoría destacan que los padres establecen normas que se refieren principalmente a la restricción temporal de su uso (Arango et al., 2010; Garitaonandia \& Garmendia, 2007; Sureda, Comas, \& Morey, 2010). No obstante, los menores perciben un escaso control en el empleo de Internet por parte de sus padres (European Commission, 2007; INTECO, 2009; Livingstone $\&$ Bober, 2006; Sotomayor, 2008) e informan de que los padres no se enteran de lo que hacen ellos en Internet (Garitaonandia \& Garmendia, 2007). Melamud et al. (2009), en una muestra de jóvenes argentinos, también concluyen que los padres tienen poco conocimiento sobre lo que sus hijos realizan en Internet y, además, subestiman los potenciales riesgos del acceso a la Red.

Algunas administraciones internacionales están trabajando en distintos planes para sensibilizar a los padres, con el fin de estimularlos a adoptar una conducta activa con sus hijos. Como afirman diversas investigaciones (Grupo de Informática de la Sociedad Argentina de
Pediatría, 2007; Livingstone, 2007; Marciales $\&$ Cabra, 2011), las familias juegan un papel importante para minimizar los peligros de Internet y potenciar las oportunidades en el empleo que hacen sus hijos. Sin embargo, los resultados de los estudios no muestran acuerdo con el papel de la mediación parental; mientras que para unos se restringen los riesgos (Rosen, Cheever, \& Carrier, 2008), otros han puesto de manifiesto que la mediación restrictiva no se relaciona con ningún tipo del uso de Internet en los adolescentes (Lee \& Chae, 2007; Valcke, de Wever, van Keer, \& Schellens, 2011). Valcke, Bonte, de Wever, y Rots (2010) explican la inconsistencia de los resultados en función del nivel cultural de los padres, y del uso que los mismos realizan de Internet. Lou, Shih, Liu, Guo, y Tseng (2010) relacionan el nivel de alfabetización digital de los padres con el control parental, siendo menor la intervención de los padres que informan de un uso alto de Internet.

Para intentar establecer cuál es el papel de la socialización familiar (modelaje, influencia de las actitudes y mediación) en el empleo de Internet de los hijos, se han establecido en esta investigación tres objetivos principales: a) conocer el nivel de uso de Internet de los adolescentes y de sus padres, así como la actitud de los padres hacia Internet y las actividades de mediación que llevan a cabo con sus hijos; b) analizar la percepción que tienen los padres del uso de Internet de sus hijos/as y la percepción que tienen los hijos/as sobre el comportamiento de mediación que realizan sus padres; c) determinar los factores de socialización familiar que mejor predicen en el empleo de Internet en los adolescentes con la finalidad de aportar datos para la intervención familiar.

\section{Método}

\section{Participantes}

De un total de 1607 familias que participaron en el estudio, 938 cuestionarios fueron completados por los estudiantes y sus padres. Participaron en la investigación ocho institutos. Fueron 
seleccionados dos centros públicos de Enseñanza Secundaria en cada una de las cuatro provincias de Castilla-La Mancha. Los adolescentes son estudiantes españoles de Enseñanza Obligatoria Secundaria de edades comprendidas entre 12 y 17 años, $M=13.38, S D=1.55$. Un $34.1 \%$ entre 12 y 13 años, un $40 \%$ entre 14 y 15 años, y un $25.6 \%$ es mayor de 15 años. El $45.4 \%$ son chicos y el $56.6 \%$ chicas. Los cuestionarios de los padres fueron completados, un $14.1 \%$ por el padre, 49.7 $\%$ por la madre, y un $35.1 \%$ de los casos, por ambos progenitores. La mayoría tiene estudios primarios, $47.4 \%$ de los padres, $49.3 \%$ de las madres. Una tercera parte ha realizado estudios secundarios, $33.6 \%$ de los padres y $32 \%$ de las madres. Un $15.8 \%$ de los padres y un $16.6 \%$ de las madres tiene titulación universitaria.

\section{Instrumentos}

Para conocer el empleo de Internet, se formularon ocho ítems que hacían referencia a la frecuencia con la que se llevan a cabo una serie de actividades cuando los adolescentes se conectan a Internet (redes sociales, e-mails, mensajería, chat, web, compras, descargas, videojuegos). Los ítems se contestaban mediante una escala de respuesta tipo Likert: $1=$ nunca, $2=$ alguna vez al mes, 3 = alguna vez a la semana, $4=$ alguna vez al día, y $5=$ varias veces al día.

La escala fue completada por los adolescentes y por sus padres. Los padres contestaban sobre su propio comportamiento y sobre el conocimiento del empleo de Internet por parte de sus hijos. En esta última escala, se añadió a las opciones de respuesta la categoría $0=$ No sabe.

El análisis factorial muestra dos factores: actividades de interacción (constituidas por los 4 primeros ítems) y actividades cerradas (constituidas por los 4 ítems finales). El primero, las actividades de interacción, explica el $36.96 \%$ de la varianza en la escala de los padres, con una fiabilidad de 0.85; en la escala de empleo de los adolescentes, explica el $30.63 \%$ de la varianza, con una fiabilidad de 0.81. El segundo factor explica el $21.8 \%$ en la escala de padres, y el 21.8
$\%$ en la de los hijos. En ambos casos la fiabilidad es de 0.6.

En la medición de la actitud hacia Internet, se empleó una escala de elaboración propia constituida por 10 ítems, con una escala de respuesta de 5 anclajes desde $1=$ Muy en desacuerdo hasta $5=$ Muy de acuerdo. El análisis factorial de la escala agrupó los ítems en dos factores: el primero agrupa siete ítems que hacen referencia a la preocupación por los aspectos negativos de Red, con una fiabilidad de 0.79. Por ejemplo, "Los jóvenes pueden encontrarse en Internet con gente que quiera hacerles daño", "Pasar mucho tiempo en Internet puede dañar la salud". El segundo factor agrupa tres ítems que se refieren a los beneficios que puede aportar el empleo de Internet y el control que se puede realizar, con una fiabilidad baja, de 0.36 , que podría justificarse por el número reducido de ítems que lo constituyen. Por ejemplo, "Internet permite acceder a la información más actual de todo el mundo", "El manejo de Internet sirve para encontrar una profesión mejor en el futuro".

Para evaluar la mediación parental, se ha construido una escala a partir de ítems extraídos de otras investigaciones (Lee \& Chae, 2007; Livingstone \& Helsper, 2008; Mesch, 2009), que hacen referencia a los tipos de reglas y prácticas que utilizan los padres en relación al uso que hacen sus hijos de Internet. La escala fue completada por los padres y por los hijos, con el objetivo de conocer su percepción sobre el comportamiento de los padres. Todos los ítems han sido formulados afirmativamente, y se ha adoptado una escala de respuesta tipo Likert ( $1=$ nunca; $4=$ siempre $)$. En total, la escala está constituida por 23 ítems que evalúan la mediación activa, por ejemplo, "Han establecido reglas sobre los sitios web que puede o no puede visitar"; la mediación restrictiva, por ejemplo, "Le revisan sus correos electrónicos", "Le permiten registrarse en sitios web"; y el uso conjunto, por ejemplo, "Participan en actividades online con él/ella". La estructura factorial diferencia tres factores que explican el $55.2 \%$ de la varianza. Se mantiene la diferencia entre mediación activa (21.39\% de la varianza, fiabilidad 0.89) y mediación restrictiva $(17.13 \%$ de la varianza, 
fiabilidad 0.89). Los ítems de uso conjunto cargan en los dos factores, pasando los ítems de interacción positiva a la mediación activa. Los ítems de permisión de uso de Internet constituyen un único factor $(16.78 \%$ de la varianza, fiabilidad de 0.84).

\section{Procedimiento}

En primer lugar, se contactó a los equipos directivos de los diferentes centros educativos, con la finalidad de explicarles los objetivos de la investigación, y proponerles su participación voluntaria. Los centros establecieron los días para la recogida de datos, con el fin de interferir lo menos posible en el ritmo de las clases. Al no superar el tiempo de administración de los cuestionarios los 60 minutos, los alumnos cumplimentaron los cuestionarios en sus respectivas aulas en horario de tutorías, de forma colectiva, y con previa autorización de los padres para su cumplimentación. Cada sobre entregado incluía dos cuadernillos, que contenían respectivamente las escalas que debían cumplimentar los alumnos y las escalas que debían cumplimentar sus padres. Cada alumno se llevaba a su casa el sobre con el cuestionario para los padres. Tras su cumplimentación, los padres lo volvían a introducir en el sobre cerrado, para mantener la confidencialidad de la información, y se lo entregaban a sus hijos que los hacían llegar a sus tutores. Los cuestionarios fueron identificados con un código para su posterior asignación familiar.

Para el primer objetivo, se han calculado los estadísticos descriptivos de cada variable, y se han comparado los datos en función del sexo y de la edad. Para el segundo objetivo, se han calculado los descriptivos de las variables de percepción, y se han analizado las relaciones entre las variables parentales y el empleo de Internet de los hijos. En ambos casos, se han realizado los contrastes mediante la prueba chicuadrado, $t$ de Student o Anova, según el tipo de variable y el número de grupos. Finalmente, mediante la regresión logística, se ha construido un modelo con aquellos factores que se han mostrado diferenciales en el empleo de Internet. El nivel de significación estadística sugerido en todas las pruebas es de $p<0.05$. El análisis estadístico de los datos se ha realizado mediante el paquete estadístico SPSS, versión 22.

\section{Resultados}

\section{Análisis descriptivos de los adolescentes}

El $98 \%$ de los estudiantes ha realizado alguna actividad en Internet. Más de la mitad de los adolescentes emplean Internet diariamente para mantener contactos a través de las redes sociales y con mensajería instantánea. La utilizan más las chicas que los chicos. El 50 \% de los chicos juega online (Tabla 1).

Tabla 1

Empleo de Internet en los adolescentes según sexo

\begin{tabular}{|c|c|c|c|c|c|c|}
\hline & Nunca & $\begin{array}{l}\text { Alguna vez } \\
\text { al mes }\end{array}$ & $\begin{array}{l}\text { Alguna vez } \\
\text { a la semana }\end{array}$ & $\begin{array}{l}\text { Alguna vez } \\
\text { al día }\end{array}$ & $\begin{array}{l}\text { Varias veces } \\
\text { al día }\end{array}$ & $X^{2}$ \\
\hline \multicolumn{7}{|l|}{ Chicos } \\
\hline Acceder a red social & 12 & 7.6 & 20.7 & 29.9 & 29.9 & $10.79^{* *}$ \\
\hline Enviar y recibir e-mails & 21.2 & 34.8 & 25 & 13 & 6 & $14.54 * *$ \\
\hline Mensajería instantánea & 21.2 & 19.8 & 27.2 & 20.9 & 10.9 & $30.65 * * *$ \\
\hline Chatear & 15 & 8.4 & 16.6 & 29.7 & 30.2 & 6.53 \\
\hline Participar en webs & 53.1 & 16.3 & 13.9 & 9.8 & 6.8 & 0.42 \\
\hline Comprar online & 62.5 & 22 & 8.2 & 4.9 & 2.4 & 5.67 \\
\hline Descargar archivos & 12 & 22.1 & 28.1 & 18.5 & 19.3 & 6.15 \\
\hline Videojuegos online & 35.2 & 17.4 & 21.5 & 13.6 & 12.2 & $45.84^{* * * *}$ \\
\hline \multicolumn{7}{|l|}{ Chicas } \\
\hline Acceder a red social & 7.4 & 7 & 17.1 & 29.3 & 39.2 & \\
\hline Enviar y recibir e-mails & 12.6 & 32.4 & 31.5 & 17.1 & 6.3 & \\
\hline Mensajería instantánea & 15.5 & 11 & 24.3 & 28.8 & 20.3 & \\
\hline Chatear & 14 & 10.2 & 16.9 & 22.8 & 36.1 & \\
\hline Participar en webs & 53.5 & 17.6 & 13.1 & 9 & 6.8 & \\
\hline Comprar online & 67 & 19.1 & 8.2 & 5 & 0.7 & \\
\hline Descargar archivos & 12.9 & 21.7 & 29.1 & 22.8 & 13.5 & \\
\hline Videojuegos online & 50.2 & 24.7 & 13.3 & 7.2 & 4.5 & \\
\hline
\end{tabular}

Para calcular la intensidad de empleo de Internet, sumados todos los ítems, el empleo es similar en los chicos, $M=21.61$, que en las chicas, $M=21.87, t=-0.58, p=0.559$. Sin embargo, las chicas realizan más actividades de interacción, $M=13.42$, que los chicos, $M=$ 12.38, $t=-3.62, p<0.001$. En las actividades cerradas se observa la relación contraria, más empleo en los chicos, $M=9.23$, que en las chicas, $M=8.45, t=3.48, p<0.001$. La correlación entre la realización de actividades de interacción $\mathrm{y}$ actividades cerradas es significativa, $r=0.45, p$ $<0.001$.

Los adolescentes informan de mayor empleo de Internet conforme se incrementa la edad, M12-13años $=20.57, M_{14-15 \text { años }}=22.04, \mathrm{M}$ 
+15 años $=22.85, F=8.56, p<0.001$, siendo la diferencia significativa tanto en las actividades de interacción, $F=8.22$, $p<0.001$, como en las actividades cerradas, $F=4.39, p<0.05$.

\section{Análisis descriptivos de los padres}

El $70 \%$ de los padres utiliza el correo electrónico (Tabla 2). Casi el $20 \%$ no realiza nunca ninguna actividad de las evaluadas en Internet. Un $25 \%$ de los padres no ha realizado nunca una actividad de interacción, un $33 \%$ no ha llevado a cabo nunca una actividad cerrada.

Tabla 2

Porcentajes empleo de Internet de los padres

\begin{tabular}{lrrrrr}
\hline & Nunca & $\begin{array}{c}\text { Alguna vez } \\
\text { al mes }\end{array}$ & $\begin{array}{r}\text { Alguna vez } \\
\text { a la semana }\end{array}$ & $\begin{array}{c}\text { Alguna vez } \\
\text { al día }\end{array}$ & \multicolumn{2}{c}{$\begin{array}{c}\text { Varias veces } \\
\text { al dia }\end{array}$} \\
\hline Acceder a red social & 59 & 12.9 & 16.1 & 10.3 & 1.8 \\
Enviar y recibir emails & 30.2 & 17.9 & 24.5 & 16.4 & 11.1 \\
Mensajería instantánea & 63.8 & 15 & 13.6 & 5.6 & 1.9 \\
Chatear & 74 & 12.5 & 9 & 3.8 & 0.8 \\
Participar en webs & 84.9 & 10.1 & 2.5 & 2 & 0.5 \\
Comprar online & 52 & 33.7 & 10.8 & 2.6 & 0.9 \\
Descargar archivos & 54.6 & 27 & 13.5 & 3 & 1.9 \\
Videojuegos online & 89.5 & 4.6 & 3.5 & 2.1 & 0.2 \\
\hline
\end{tabular}

Sumados todos los ítems, para calcular la intensidad de empleo de Internet, se comprueba su bajo uso en los padres. La media de empleo es de 13.26, siendo ligeramente superior en las actividades de interacción, $M=7.52$, que en las actividades cerradas, $M=5.77, t=17.53, p$ $<0.001$. La correlación entre la realización de actividades de interacción y actividades cerradas es significativa, $r=0.61, p<0.001$.

En los descriptivos de la actitud hacia Internet, predomina la preocupación por los aspectos negativos, $M=4.11$, sobre los beneficios que podría aportar a sus hijos, $M=3.94, t=5.75$, $p<0.001$. La preocupación es superior en los padres que no emplean la red, $M=4.23, M$ $=4.09, t=2.25, p<0.05$. No se encuentran diferencias significativas según el sexo del hijo. En función de la edad, no se encuentra diferencia en la actitud sobre los aspectos positivos, pero los negativos se reducen ligeramente en los padres de los adolescentes más mayores, $M_{12-13 \text { años }}=4.16$, $M_{14-15 \text { años }}=4.12, M_{+15 \text { años }}=4.02, F=3.63$, $p<0.05$.

Respecto a las estrategias de mediación parental, más del $90 \%$ de los padres hablan con sus hijos sobre los riesgos y los beneficios de Internet, más del $85 \%$ restringen y controlan el tiempo que sus hijos están conectados y el $80 \%$ están con su hijo o cerca de él mientras emplea Internet. El $89 \%$ permite que sus hijos empleen el correo electrónico, cerca del 83 \% les permiten el uso de la mensajería instantánea y chatear (Tabla 3).

\section{Tabla 3}

Empleo de las estrategias de mediación, en porcentajes

\begin{tabular}{|c|c|c|c|c|}
\hline & Nunca & Alguna vez & A menudo & Siempre \\
\hline Controlan las páginas web que visita su hijo/a & 22.5 & 26.2 & 30.7 & \\
\hline $\begin{array}{l}\text { Han establecido reglas sobre los sitios web que puede o } \\
\text { no puede visitar }\end{array}$ & 23.1 & 21.9 & 17.7 & 37.3 \\
\hline $\begin{array}{l}\text { Han establecido reglas sobre el tipo de información } \\
\text { personal que puede o no puede compartir }\end{array}$ & 14.8 & 19.3 & 20.1 & 45.7 \\
\hline Han limitado el tiempo que puede utilizar Internet & 15 & 19.5 & 32.3 & 33.1 \\
\hline Controlan el tiempo que está en Internet & 12.6 & 16.9 & 33.4 & 37.1 \\
\hline Le recomiendan sitios web para visitar & 33.3 & 36.2 & 19.4 & 11.1 \\
\hline Están & 15.6 & 32.5 & 35.6 & 16.3 \\
\hline nnectado & 12 & 34 & 33.8 & 19.9 \\
\hline Le ayud & 16.4 & 32.8 & 30.6 & 20.2 \\
\hline o'́a sobre los riesgos de Internet & 6.5 & 13.5 & 26.7 & 53.3 \\
\hline $\begin{array}{l}\text { lablan con su hijo/a sobre los beneficios de utilizar } \\
\text { Internet }\end{array}$ & 9.2 & 26.5 & 33.5 & 30.8 \\
\hline Le permiten compartir información personal & 57.9 & 26.9 & 9.3 & \\
\hline Lep & 85.1 & 11 & 1.6 & 1.9 \\
\hline & & & & \\
\hline Lep & & 33 & 28.1 & 27.2 \\
\hline Le per & 17.5 & 32.2 & 29 & 21.2 \\
\hline Le p & 37.2 & 32.9 & 18 & 11.9 \\
\hline Le per & 19.4 & 44.3 & 22.1 & 14.2 \\
\hline Le perm & 17.7 & 34.9 & 26.8 & 20.6 \\
\hline $\begin{array}{l}\text { Vigilan y supervisan los programas que instala en su } \\
\text { ordenador }\end{array}$ & 15.8 & 21.3 & 25.1 & 37.8 \\
\hline & 5 & 28.6 & 11.9 & \\
\hline Participan en actividades online con él/ella & 68. & 24. & 6.3 & 5 \\
\hline $\begin{array}{l}\text { Han instalado un programa que le impide acceder a } \\
\text { determinadas páginas web o descargar programas y } \\
\text { otros archivos }\end{array}$ & 68.5 & 12.7 & 5.5 & 13.3 \\
\hline
\end{tabular}

Predomina la mediación restrictiva, $M=3.28$, sobre la mediación activa, $M=2.34$, el nivel de permisión también es elevado, $M=3.08$. No se encuentra diferencia según el sexo del hijo en ninguno de los factores de mediación. Según la edad, solamente alcanza significación estadística el empleo de la mediación activa, que es más reducida en los padres de los adolescentes más mayores, $M_{12-13 \text { años }}=2.49, M_{14-15 \text { años }}=2.35, M$ +15 años $=2.14, F=17.72, p<0.001$. Los padres que emplean Internet emplean en mayor medida la mediación activa con sus hijos, $M=1.94, M$ $=2.42, t=-8.3, p<0.001$.

\section{Relación padres, hijos e Internet}

En el empleo de Internet que informan los padres que realizan sus hijos, predomina el uso de las redes sociales (Tabla 4). Perciben en sus hijos el mayor empleo en las actividades de interacción, $M=11.78$, que en las actividades cerradas, $M=$ $7, t=31.35, p<0.001$. 
Tabla 4

Empleo que realizan los adolescentes según sus padres

\begin{tabular}{lrrrrrr}
\hline & $\begin{array}{c}\text { No } \\
\text { sabe }\end{array}$ & Nunca & $\begin{array}{c}\text { Alguna vez } \\
\text { al mes }\end{array}$ & $\begin{array}{r}\text { Alguna vez } \\
\text { a la semana }\end{array}$ & $\begin{array}{c}\text { Alguna vez } \\
\text { al día }\end{array}$ & $\begin{array}{c}\text { Varias veces } \\
\text { al día }\end{array}$ \\
\hline Acceder a red social & 4 & 9 & 10 & 26 & 31.5 & 19.6 \\
Enviar y recibir emails & 4.9 & 14 & 25.2 & 30.3 & 17.4 & 8.2 \\
Mensajería instantánea & 7.5 & 24.4 & 18.4 & 24.8 & 15.9 & 9 \\
Chatear & 5.1 & 15.1 & 12.7 & 24.7 & 23.6 & 18.8 \\
Participar en webs & 12.7 & 51.3 & 14.1 & 11.3 & 7.4 & 3.2 \\
Comprar online & 11.4 & 61.5 & 16.1 & 7.5 & 2.4 & 1.1 \\
Descargar archivos & 4.8 & 19 & 32.6 & 26.2 & 11.7 & 5.8 \\
Videojuegos online & 10.7 & 47.3 & 16.7 & 14.4 & 7.4 & 3.5 \\
\hline
\end{tabular}

En el empleo total que informan que realizan los hijos de Internet, no se encuentran diferencias significativas según el sexo de los hijos, $\mathrm{M}_{\text {chicos }}=$ $18.94, M_{\text {chicas }}=18.63, t=0.61, p=0.541$, pero sí aparecen diferencias en el tipo de actividad que realizan, siendo superiores las actividades de interacción que informan los padres de las chicas, $\mathrm{M}_{\text {chicos }}=11.38, M_{\text {chicas }}=12.12, t=-2.2, p$ $<0.001$, predominando las actividades cerradas que informan los padres de los chicos, $\mathrm{M}_{\text {chicos }}=$ $7.5, M_{\text {chicas }}=6.58, t=4.01, p<0.001$. Respecto a la edad, los padres de los adolescentes perciben un empleo superior conforme se incrementa la edad de sus hijos, $M_{12-13 \text { años }}=17.23, M_{14-15 \text { años }}$ $=19.3, M_{+15 \text { años }}=20.03, F=10.83, p<0.001$.

Aunque la mayoría de los padres se consideran informados, su percepción está sesgada, ya que perciben un empleo más reducido del que realmente hacen sus hijos. Todas las contingencias indican diferencia entre la percepción de los padres y el empleo que informan los hijos que realizan (Tabla 5).

\section{Tabla 5}

Contingencia empleo Internet

\begin{tabular}{lcccc}
\hline & \multicolumn{2}{c}{$\begin{array}{c}\text { Empleo padres/ } \\
\text { empleo hijos }\end{array}$} & \multicolumn{2}{c}{$\begin{array}{c}\text { Información padres/ } \\
\text { empleo hijos }\end{array}$} \\
\cline { 2 - 5 } & $x^{2}$ & $p$ & $x^{2}$ & $p$ \\
\hline Acceder a red social & 31.75 & 0.011 & 515.31 & 0.000 \\
Enviar y recibir emails & 24.16 & 0.086 & 188.47 & 0.000 \\
Mensajería instantánea & 15.57 & 0.484 & 144.15 & 0.000 \\
Chatear & 19.77 & 0.231 & 364.37 & 0.000 \\
Participar en webs & 21.73 & 0.152 & 155.61 & 0.000 \\
Comprar online & 30.52 & 0.014 & 208.15 & 0.000 \\
Descargar archivos & 65.15 & 0.000 & 216.25 & 0.000 \\
Videojuegos online & 25.65 & 0.055 & 220.6 & 0.000 \\
\hline
\end{tabular}

Existe correlación significativa entre el empleo de Internet que realizan los padres y los hijos, $r=0.15, p<0.001$. El uso es similar en correo electrónico, mensajería instantánea, chat, Youtube y videojuegos online (Tabla 5).
Los adolescentes cuyos padres utilizan Internet realizan un uso más elevado, $M=22.28$, que los hijos de las familias que no acceden a la red, $M$ $=19.9, t=-4.17, p<0.001$. La actitud de los padres ante Internet no influye en el empleo que realizan los hijos, ni los aspectos positivos, $F=$ $1.34, p=0.261$, ni los aspectos negativos, $F=$ $1.43, p=0.24$.

Respecto a la mediación parental, los hijos perciben menor mediación que la que informan sus padres, tanto en la mediación activa, $M_{\text {padres }}$ $=2.34, M_{\text {hijos }}=1.89, t=19.75, p<0.001$, como en la mediación restrictiva, $M_{\text {padres }}=3.28$, $M_{\text {hijos }}=2.12, t=36.01, p<0.001$, también consideran que es más baja la permisión, $M_{\text {padres }}$ $=3.08, M_{\text {hijos }}=2.66, t=15.14, p<0.001$.

La mediación parental incide sobre el empleo de Internet de los hijos, mediando el uso que los propios padres realizan de Internet. Así, en relación con los padres que emplean Internet, las medidas de mediación que informan que realizan son atendidas por sus hijos cuando emplean estrategias activas; sin embargo, las estrategias restrictivas que llevan a cabo, parece que no suelen ser tenidas en cuenta por sus hijos. Cuando los padres no emplean Internet, la mediación activa y restrictiva que informan que realizan, influye en la reducción de las actividades de Internet en sus hijos. La percepción de mediación de los hijos solamente es sensible a la permisión de los padres (Tabla 6).

\section{Tabla 6}

Empleo de Internet de los adolescentes según los niveles de mediación paterna

\begin{tabular}{lccccc}
\hline \multicolumn{1}{c}{ Nivel de mediación paterna } & Bajo & Medio & Alto & F & $p$ \\
\hline $\begin{array}{l}\text { Padres que no emplean Internet } \\
\text { Informada por los padres }\end{array}$ & & & & & \\
Mediación activa & 18.16 & 21.38 & 18.87 & 3.31 & 0.04 \\
Mediación restrictiva & - & 22.28 & 19.05 & 6.88 & 0.01 \\
Permisión & - & 18.47 & 20.81 & 3.59 & 0.06 \\
Percibida por los hijos & & & & & \\
Mediación activa & 19.59 & 20.57 & 18.67 & 0.47 & 0.622 \\
Mediación restrictiva & 19.26 & 21.22 & 19.1 & 1.69 & 0.188 \\
Permisión & 17.5 & 19.89 & 22.37 & 4.21 & 0.017 \\
\hline Padres que emplean Internet & & & & & \\
Informada por los padres & & & & & \\
Mediación activa & 24.02 & 22.52 & 20.81 & 4.76 & 0.009 \\
Mediación restrictiva & - & 22.35 & 22.3 & 0.01 & 0.944 \\
Permisión & - & 22.06 & 22.38 & 0.33 & 0.564 \\
Percibida por los hijos & & & & & \\
Mediación activa & 23.22 & 22.19 & 18.5 & 7.46 & 0.001 \\
Mediación restrictiva & 22.84 & 22.67 & 20.52 & 6.18 & 0.002 \\
Permisión & 17.53 & 20.74 & 24.85 & 48.97 & 0.000 \\
\hline
\end{tabular}


En relación con nuestro tercer objetivo, para conocer el peso de las variables de socialización familiar en el empleo de Internet de los hijos, se llevó a cabo un análisis de regresión. De las variables de estudio, entran a formar parte de la ecuación como predictores del empleo de Internet en los adolescentes: el empleo de Internet de los padres, $\beta=0.13, t=2.43, p$ $<0.05$, las estrategias de mediación activa, $\beta=$ -0.93, $t=-2.01, p<0.05$, y la percepción de permisión de empleo de Internet que tienen los hijos, $\beta=3.43, t=9.17, p<0.001$. El modelo muestra buen ajuste global, $F=16.65, p<0.001$. El coeficiente $\mathrm{R}^{2}$ de Nagelkerke indica que el $17 \%$ de la variabilidad del uso de Internet de los adolescentes viene explicada por factores de socialización familiar.

\section{Discusión}

El objetivo de este trabajo fue analizar la influencia de las variables de socialización familiar en Internet, en relación con el empleo de la red que llevan a cabo los hijos. Para ello, en primer lugar, se llevó a cabo un análisis descriptivo del uso de Internet de padres e hijos. También se analizaron la actitud ante Internet y las estrategias de mediación de los padres, junto con la percepción del uso que realizaban sus hijos. Consideramos también importante conocer la percepción de los hijos sobre las medidas que adoptan sus padres respecto al control del empleo de Internet.

Los resultados confirman que un elevado porcentaje de adolescentes, $98 \%$, realiza actividades en la red. Porcentaje ligeramente superior al informado en estudios previos realizados en el contexto internacional (Almansa et al., 2013; García et al., 2013; Hernández et al., 2009; Valkenburg \& Peter, 2007). La intensidad de empleo es similar en chicos y en chicas (Viñas, 2009), pero utilizan diferentes recursos. Se reproduce un patrón diferencial según el sexo. Las chicas, básicamente, para mantener relaciones sociales (Arango et al., 2010; Costa, 2011; De Haro, 2010; Flores, 2009; Herrera-Batista, 2009; Notley, 2009), y los chicos utilizan más juegos online (Arango et al., 2010; Fernández-Montalvo et al., 2014).

Los padres informan de bajo uso de Internet, considerando que un $20 \%$ nunca ha tenido acceso. Los que lo emplean, lo utilizan fundamentalmente, para acceder al correo electrónico (70 \%). Resulta interesante destacar que se correlacionan las medidas de empleo de Internet de padres e hijos. Sin embargo, no influye la actitud de los padres hacia Internet.

Diversos estudios han señalado la preocupación de los padres por Internet (Echeburrúa et al., 2009; Parra, 2010), y nuestros resultados indican que existe una mayor preocupación de los padres por los aspectos negativos de la red, superior en los padres que no la utilizan, aunque esta preocupación se va reduciendo conforme se incrementa la edad de los hijos.

Respecto a las medidas de mediación, otros estudios habían indicado la superioridad de la restricción temporal (Arango et al., 2010; Garitaonandia \& Garmendia, 2007; Sureda et al., 2010). En nuestro estudio, las medidas de mediación están correlacionadas, los padres emplean conjuntamente mediación activa y mediación restrictiva, aunque los padres que emplean Internet se dirigen más a actividades de mediación activa. No existe diferencia según el sexo de los hijos, pero conforme se incrementa la edad la mediación parental, se va reduciendo. Los padres permiten a sus hijos emplear el correo electrónico y chatear con los amigos. Coincidiendo con investigaciones anteriores (Inteco, 2009; Livingstone \& Bober, 2006; Sotomayor, 2008), los menores no perciben la mediación de sus padres, pero tampoco son concordantes en la percepción de permisión que informan sus padres. Existe desacuerdo sobre la influencia de la mediación parental sobre el comportamiento de los hijos. Los resultados obtenidos nos llevan a pensar que la acción de los padres está mediada por el empleo que realizan de Internet. Las estrategias restrictivas, cuando son empleadas por los padres que usan Internet no son tenidas en cuenta por los hijos. Estos responden mejor a las estrategias activas de mediación. 
Estudios previos habían concluido que los padres no se enteran de lo que hacen sus hijos en Internet (Garitaonandia \& Garmendia, 2007; Melamud et al., 2009). Sin embargo, en nuestro estudio los padres sí conocen las actividades que realizan sus hijos. Los padres de las chicas indican en mayor medida actividades de relaciones sociales, y los padres de los chicos indican los juegos online. Aunque, en todos los casos, perciben un empleo menor al que han informado sus hijos.

El análisis de regresión confirma la importancia de las variables perceptivas de los hijos, destacando el modelaje que realizan los padres y la permisión en el uso de Internet que perciben. También resulta significativa la mediación activa. Los resultados obtenidos señalan la relevancia del papel de los padres en la socialización del uso de internet, confirmando la mediación del empleo que realizan los padres (Valcke et al., 2010; Lou et al., 2010). Como afirman Dehue et al. (2008), es imprescindible que los padres colaboren en los procesos de socialización de los nuevos medios.

La representatividad de la muestra es elevada ya que fue tomada en la propia aula durante un periodo de escolaridad que es obligatoria para los adolescentes españoles. Sin embargo, resulta difícil generalizar los resultados ya que la muestra procede únicamente de institutos españoles.

Para futuras líneas de investigación, sería interesante replicar este tipo de trabajos con muestras más amplias y de diferentes contextos para profundizar en las variables de protección y riesgo familiar. Es importante hacer hincapié en el uso adecuado de Internet en los adolescentes para evitar una problemática posterior o una dependencia futura al mismo (Ruiz-Olivares et al., 2010), y potenciar los efectos positivos.

También se considera de interés, indagar con mayor profundidad en el empleo y el conocimiento de Internet por los padres, para poder programar acciones de formación que les facilite ayudar a sus hijos en la relación positiva con Internet y reducir la preocupación por la Red.

En el sentido positivo de la influencia de las TIC, el desarrollo de buenas prácticas en Internet puede conducir a los estudiantes a obtener mejores logros académicos (Chen \& Fu, 2009; Gil-Flores, 2009; Leung \& Lee, 2012; Torres et al., 2016). Son jóvenes que no han conocido el mundo sin Internet, y para los cuales las tecnologías digitales son mediadoras de gran parte de sus experiencias (UNESCO, 2013, p. 14). Debemos aprovechar esta experiencia de los jóvenes para trasladarla a las aulas.

Los cambios sociales que ha provocado la tecnología incluyen también el ámbito educativo, tanto en el aprendizaje como en la metodología. En la mayoría de los centros educativos se han incorporado las TIC: pizarras digitales, grupos de WhatsApp, salas de informática, tablets, etc. Todo ello exige a los estudiantes y a los docentes, las habilidades y capacidades necesarias para acceder a su empleo. También sería de gran interés analizar el conocimiento, las competencias y el empleo de Internet que tienen los docentes. Es preciso que ellos también presenten un adecuado desarrollo para implementar efectivamente las TIC en la escuela, e incrementar su empleo para aumentar la motivación y el rendimiento de los escolares. Más relevante, si cabe, es la incorporación en la educación superior. La infraestructura tecnológica ha modificado la generación y la difusión del conocimiento. No podemos dejar de lado, que será el contexto inmediato de acceso para muchos de los alumnos de nuestro estudio.

Con independencia de la dirección que tomen de futuro, como afirman Casillas, RamírezMartinell, y Ortega-Guerrero (2016, p. 156), deberán incorporarse "en un mundo laboral rodeado de TIC": ambientes virtuales, bases de datos, recursos tecnológicos, programas informáticos y dispositivos digitales.

\section{Agradecimientos}

Esta investigación se ha realizado gracias a la financiación concedida por el Ministerio de Economía y Competitividad de España dentro del Programa Nacional de Investigación e Innovación al proyecto con referencia PSI2015-70822-R 


\section{Referencias}

Almansa, A., Fonseca, O., \& Castillo, A. (2013). Redes sociales y jóvenes. Uso de Facebook en la juventud colombiana y española. Comunicar, 20(40), 127-135. https://doi.or g/10.3916/C40-2013-03-03

Arango, G., Bringué, X., \& Sádaba, C. (2010). La generación interactiva en Colombia: adolescentes frente a la Internet, el celular y los videojuegos. Anagramas. Rumbos y Sentidos de la Comunicación, 9(17), 45-56. Recuperado de http://www.scielo.org.co/scielo.php?scri pt $=$ sci_arttext\&pid $=$ S1692-25222010000 200004

Bringué, X., Sádaba, C., \& Tolsa, J. (2011). La generación interactiva en Iberoamérica 2010. Niños y adolescentes antes las pantallas. Madrid: Foro Generaciones Interactivas.

Casillas, M. A., Ramírez-Martinell, A., \& Ortega-Guerrero, J. C. (2016). Afinidad tecnológica de los estudiantes universitarios. Innovación Educativa, 16(70), 151-175.

Castellana, M., Sánchez-Carbonell, X., Graner, C., \& Beranuy, M. (2007). El adolescente ante las tecnologías de la información y la comunicación: Internet, móvil y videojuegos. Papeles del Psicólogo, 28(3), 196-204. Recuperado de http://www.papel esdelpsicologo.es/vernumero.asp?id $=1503$

Chen, S. Y., \& Fu, Y. C. (2009). Internet use and academic achievement: gender, differences in early adolescente. Adolescence, 44(176), 797-812.

Costa, J. (2011). Los jóvenes portugueses de los 10 a los 19 años: iqué hacen con los ordenadores?. Teoría de la Educación. Educación y Cultura en la Sociedad de la Información, 12(1), 209-239.

Dehue, F., Bolman, C., \& Völlink, T. (2008). Cyberbullying: Youngsterrs' experiences and parental perception. CyberPsychology and Behavior, 11(2), 41-47. https://doi.org/ 10.1089/cpb.2007.0008

De Haro, J. J. (2010). Redes sociales para la educación. Madrid: Anaya.
Echeburrúa, E., Labrador, F. J., \& Becoña, E. (Eds.). (2009). Adicción a las nuevas tecnologías en adolescentes y jóvenes. Madrid: Pirámide.

Estévez, L., Bayón, C., de la Cruz, J., \& Fernández-Liria, A. (2009). Uso y abuso de Internet en adolescentes. En E. Echeburrúa, F. J. Labrador, \& E. Becoña (Eds.), Adicción a las nuevas tecnologías en adolescentes y jóvenes (pp. 101-128). Madrid: Pirámide.

European Commission. (2007). Safer Internet for children. Qualitative study in 29 European countries. Recuperado de http://ec.europa.eu/ information_society/activities/sip/docs/

eurobarometer/qualitative_study__2007/ summary_report_en.pdf

Fernández-Montalvo, J., Peñalva, A., \& Irazabal, I. (2014). Hábitos de uso y conductas de riesgo en Internet en la preadolescencia. Comunicar, 22 (44), 113-120. https://doi.or $\mathrm{g} / 10.3916 / \mathrm{C} 44-2015-12$

Flores, J. M. (2009). Nuevos modelos de comunicación, perfiles y tendencias en las redes sociales. Comunicar, 17(33), 73-81. h ttps://doi.org/10.3916/c33-2009-02-007

Fosk, A. (2010). Situación de Internet en Latinoamérica. Recuperado de http://www.s lideshare.net/francoalfero/estado-de-intern et-en-latinoamerica-comscore-2010

García, A., López de Ayala, M. C., \& Catalina, B. (2013). Hábitos de uso de Internet y en las redes sociales de los adolescentes españoles. Comunicar, 21 (41), 195-204. https://doi.or $\mathrm{g} / 10.3916 / \mathrm{C} 41-2013-19$

García-Piña, C. A. (2008). Riesgos del uso de Internet por niños y adolescentes. Estrategias de seguridad. Acta Pediátrica de México, 29(5), 273-279. Recuperado de http://www.imbiomed.com.mx/1/1/artic ulos.php?method=showDetail\&id_articul $\mathrm{o}=53603$ \&id_seccion $=3348$ \&id_ejempla $\mathrm{r}=5424 \&$ \&__revista $=17$

Garitaonandia, C., \& Garmendia, M. (2007). Cómo usan Internet los jóvenes: hábitos, riesgos y control parental. Recuperado 
de http://www.anobium.es/docs/gc_fichas/ doc/01237BEXqx.pdf

Gil-Flores, J. (2009). Computer use and students' academic achievement. Research, reflections and innovations in integrating ICT in education (Internet). Recuperado de http://g oo.gl/ODCcGT

Grupo de Informática de la Sociedad Argentina de Pediatría. (2007). Los niños, sus padres, Internet y los pediatras. Archivos Argentinos de Pediatría, 105(4), 368-371. Recuperado de http://www.scielo.org.ar/scielo.php?pid $=$ S0325-00752007000400015\&script $=$ sci arttext

Hernández, C., Robles, E., \& Mella, J. (2009). Medición de la actitud al uso del computador e Internet en estudiantes de enseñanza media en Santiago de Chile. Nuevas Ideas en Informática Educativa, 5, 28-35. Recuperado de http://www.tise.cl/20 09/tise_2009/pdf/4.pdf

Herrera-Batista, M. A. (2009). Disponibilidad, uso y apropiación de las tecnologías por estudiantes universitarios en México: perspectivas para la incorporación innovadora. Revista Iberoamericana de Educación, 48(6), 1-19. Recuperado de htt p://www.rieoei.org/2630.htm

Heyam, A. (2014). The influence of Social Networks on students' performance. Journal of Emerging Trends in Computing and Information Sciences, 5(3), 200-205.

Holtz, P., \& Appel, M. (2011). Internet use and video gaming predict problem behavior in early adolescence. Journal of Adolescence, 34(1), 49-58. https://doi.org/10.1016/j.adol escence.2010.02.004

Instituto Nacional de Tecnologías de la Comunicación, INTECO. (2009). Estudio sobre los hábitos seguros en el uso de las TIC por niños y adolescentes y e-confianza de sus padres. Madrid: Instituto Nacional de Tecnologías de la Comunicación.

Lee, S. J., \& Chae. Y. W. (2007). Children's Internet use in a family context: Influence on family relationships and parental mediation. CyberPsychology $\mathscr{G}$ Behavior,
10(5), 640-644. https://doi.org/10.1089/cp b.2007.9975

Leung, L., \& Lee, P. (2012). Impact of Internet literacy, Internet addiction symptoms and Internet activities on academic performance. Social Science Computer Review, 30(4), 403-418. https://doi.org/10.1 $177 / 0894439311435217$

Livingstone, S. (2007). Los niños en Europa. Evaluación de los riesgos de Internet. Telos, 73. Recuperado de http://telos.fundaciontelefonica.com/tel os/articulocuaderno.asp@idarticulo=2\&r $\mathrm{ev}=73 . \mathrm{htm}$

Livingstone, S., \& Bober, M. (2006). Regulating the Internet at home: Contrasting the perspectives of children and parents. En D. Buckingham, \& R. Willet (Eds.), Digital generations. Children, young people, and new media (pp. 93-114). Londres: Rouledge.

Livingstone, S., \& Helsper, E. J. (2008). Parental mediation of children's Internet use. Journal of Broadcasting $\mathcal{E}$ Electronic Media, 52(4), 581-599. https://doi.org/10.1080/08838150 802437396

Lou, S. J., Shih, R. C., Liu, H. T., Guo, Y. C. , \& Tseng, K. H. (2010). The influences of the sixth graders' parents' Internet literacy and parenting style on Internet parenting. TOJET: Turkih Online Journal of Educational Technology, 9(4), 173-184. Recuperado de h ttp://www.tojet.net/articles/v9i4/9417.pdf

Marciales, G. P., \& Cabra, F. (2011). Internet y pánico moral: revisión de la investigación sobre la interacción de niños y jóvenes con los nuevos medios. Universitas Psychologica, 10(3), 855-865. Recuperado de http://revistas.javeriana.edu.co/index.p $\mathrm{hp} / \mathrm{revP}$ sycho/article/view/841

Melamud, A., Nasanovsky, J., Otero, P., Canosa, D., Enríquez, D., Köhler, C...\& Svetliza, J. (2009). Usos de Internet en hogares con niños entre 4 y 18 años. Control de los padres sobre este uso. Resultados de una encuesta nacional. Archivos Argentinos de Pediatría, 107(1), 30-36.

Mendivelso, L., Méndez, J. M., \& RamírezMartinell, A. (2017). Integración de un 
modelo de aprendizaje innovador como apoyo TIC en el área del lenguaje. Revista de Investigación Educativa de la Escuela de Graduados en Educación, 7(14). Recuperado de http://rieege.tecvirtual.mx/

Mesh, G. S. (2009). Parental mediation, online activities, and cyberbullying. CyberPsychology $\mathfrak{G}$ Behavior, 12(4), 387-393. https://doi.org/10.1089/cpb.2009. 0068

Notley, T. (2009). Young people, online networks, and social inclusion. Journal of ComputerMediated Communication, 14, 1208-1227. doi: 10.1111/j.1083-6101.2009.01487.x

Osma, J. (2002). Observatorio epidemiológico de sustancias psicoactivas, uso de Internet y juegos electrónicos en una institución universitaria. Adicciones, 14(2), 209-225.

Parra, E. (2010). Las redes sociales de Internet: también dentro de los hábitos de los estudiantes universitarios. Tendencias, 6(2), 193-207. Recuperado de http://www.google.es/url?sa $=\mathrm{t} \& \mathrm{rct}=\mathrm{j} \&$ $\mathrm{q}=$ \&esrc $=\mathrm{s} \&$ source $=$ web\&cd $=1 \& \mathrm{ved}=$ OCB4QFjAAahUKEwjQm9_JtvzIAhVJ2B oKHYYLA5Q\&url=http\%3A\%2F\%2Fdia lnet.unirioja.es\%2Fdescarga $\% 2$ Farticulo\% 2F3640635.pdf\&usg =AFQjCNEeIDYGev PSeWf44EcG9eezM8E51Q

Rosen, L. D., Cheever, N. A., \& Carrier, M. L. (2008). The impact of parental attachment style, limit setting and monitoring on teen MySpace behavior. Journal of Applied Developmental Psychology, 29, 459-471.

Ruiz-Olivares, R., Lucena, V., Pino, M. J., \& Herruzo, J. (2010). Análisis de comportamientos relacionados con el uso/ abuso de Internet, teléfono móvil, compras y juego en estudiantes universitarios. Adicciones, 22(4), 301-310. Recuperado de http://www.redalyc.org/articulo.oa?id= 289122896003

Schwarz, O. (2011). Who moved my conversation? Instant messaging. Intertextuality and new regimes of intimacy and truth. Media Culture Society, 33(1), 71-87.
Sotomayor, E. (2008). Normas y pautas de uso de Internet de los adolescentes y jóvenes españoles. En J. F. Tezanos (Ed.), Internet y las familias (pp. 107-142). Madrid: Síntesis.

Sureda, J., Comas, R., \& Morey, M. (2010). Menores y acceso a Internet en el hogar: las normas familiares. Comunicar, 17(34), 135-143. https://doi.org/10.3916/C34-2010 $-03-13$

Thurlow, C., \& McKay, S. (2003). Profiling 'new' communication technologies in adolescence. Journal of Language and Social Psychology, 22(1), 94-103. https://doi.org/1 0.1177/0261927X02250060

Torres, J., Duan, J., Gómez-Alvarado, H. F., Marín-Gutiérrez, I., \& Segarra, V. (2016). Usos de Internet y éxito académico en estudiantes universitarios. Comunicar, 24(48), 61-70. https://doi.org/10.3916/C48 $-2016-06$

UNESCO. (2013) Enfoques estratégicos sobre las TIC en educación en América Latina y el Caribe. París: UNESCO, Oficina Regional de Educación para América Latina y el Caribe.

Valcke, M., de Wever, B., van Keer, H., \& Schellens, T. (2011). Long-term study of safe Internet use of young children. Computers \&8 Education, 57(1), 1292-1305. https://doi.org/10.1016/j.comp edu.2011.01.010

Valcke, M., Bonte, S., de Wever, B., \& Rots, I. (2010). Internet parenting styles and the impact use of primary school children. Computers $\mathscr{E}$ Education, 55(2), 454-464. https://doi.org/10.1016/j.comped u.2010.02.009

Valkenburg, P. M., \& Peter, J. (2007). Preadolescents' and adolescents' online communication and their closeness to friends. Developmental Psychology, 43(2), 267-277. https://doi.org/10.1037/0012-164 9.43.2.267

Viñas, F. (2009). Uso autoinformado de Internet en adolescentes: perfil psicológico de un uso elevado de la red. International Journal of Psychology and Psychological Therapy, 9(1), 109-122. 


\section{Notas}

* Artículo de investigación. 